\title{
Launching the CUSBEA Article Series in SCIENCE CHINA Life Sciences
}

As a CUSBEA (China-United States Biochemistry Examination and Administration) Program fellow of Class IV (1985), I am very excited to announce the official launch of the CUSBEA Article Series in SCIENCE CHINA Life Sciences, a journal in which I am currently serving as Executive Vice-Editor-in-Chief. A couple of months ago, I initially proposed this idea to the Editor-in-Chief of SCIENCE CHINA Life Sciences, Professor Wang Da-Cheng and to the Editor General of SCIENCE CHINA Life Sciences, Professor Zhu Zuoyan, both of whom responded very positively. The article contributed by Dr. Luo Liqun [1], CUSBEA fellow of Class VI (1987) and currently professor in the Department of Biology at Stanford University, as well as Investigator at the Howard Hughes Medical Institute, marks the official beginning of this series.

Three previously published SCIENCE CHINA Life Sciences articles might also be considered as part of this series. Dr. Shi Yang, who was a CUSBEA fellow of Class II (1983) and is currently professor of Pediatrics at Harvard Medical School, contributed one article in this series [2]. I also contributed two articles [3,4]. Science China Series C-Life Sciences published the February 2009 issue as a special issue in memory of Professor Ray Wu, primary founder and guiding leader of the CUSBEA Program [5].

The CUSBEA Program operated from 1982-1989 with a total of 422 fellows selected by means of a very competitive examination and interview process [6-8]. Many of those who continued their careers in academia are making a remarkable impact in the field of Life Sciences, mostly working in China or in the United States [7]. This special CUSBEA series aims to publish a variety of articles related to the CUSBEA program. These articles may include reviews and research articles written by former CUSBEA fellows. However, recollections from anyone concerning anything related to the CUSBEA program will also be highly welcomed. This CUSBEA series of articles will intermittently appear in the journal. Each article in this special series will be accompanied with a photo, as well as a brief introduction of the major contributor. We encourage all former CUSBEA fellows and those who were involved in this program in a certain capacity (e.g., professors who participated in the selection process or those who mentored CUSBEA fellows) to contribute to this special series.

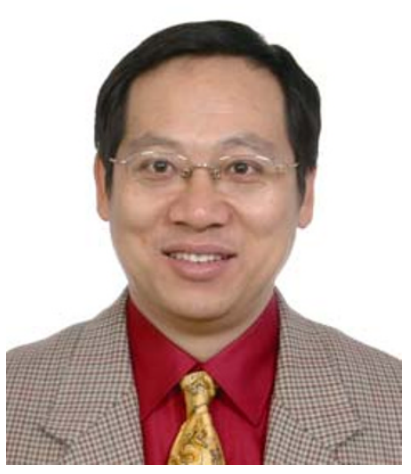

CHANG ZengYi

School of Life Sciences, Peking University, Beijing 100871, China
1 Liang L, Luo L Q. The olfactory circuit of the fruit fly Drosophila melanogaster. Sci China Life Sci, 2010, 53: 472-484

2 Lan F, Shi Y. Epigenetic regulation: Methylation of histone and non-histone proteins. Sci China C Life Sci, 2009, 52: 311-322

3 Feng Y J, Zhang M, Hu M X, et al. Disassembly intermediates of $\mathrm{RbsD}$ protein remain oligomeric despite the loss of an intact secondary structure. Sci China C Life Sci, 2009, 52: 997-1002

4 Chang Z. Posttranslational modulation on the biological activities of molecular chaperones. Sci China C Life Sci, 2009, 52: 515-
520

5 Jiang C Y. Commemorating the life of a legend. Sci China C Life Sci, 2009, 52: 97-98

$6 \mathrm{Gu}$ X C. Ray Wu and the CUSBEA Program. Sci China C Life Sci, 2009, 52: 125-127

7 Chang Z Y. The CUSBEA Program: Twenty years after. IUBMB Life, 2009, 61: 555-565

$8 \mathrm{Yu}$ R, Wu R. When east meets west: A brief history of the CUSBEA and CUSBA Programs. ASBMB Today, 2006, March issue, 8-9 